\title{
Formation Control of Mobile Robots
}

\author{
Dang Binh Nguyen, Khac Duc Do
}

\begin{abstract}
A constructive method is presented to design cooperative controllers that force a group of $N$ mobile robots to achieve a particular formation in terms of shape and orientation while avoiding collisions between themselves. The control development is based on new local potential functions, which attain the minimum value when the desired formation is achieved, and are equal to infinity when a collision occurs. The proposed controller development is also extended to formation control of nonholonomic mobile robots.
\end{abstract}

Keywords: Formation control, mobile robot, local potential function, nonholonomic mobile robot.

\section{Introduction}

Over the last few years, formation control of multiple vehicles has received a lot of attention from the control community. Applications of vehicle formation control include the coordination of multiple robots, unmanned air/ocean vehicles, satellites, aircraft and spacecraft [1]-[28]. For example, a cooperative mobile sensor network, where each mobile robot serves as a mobile sensor, is expected to outperform a single large vehicle with multiple sensors or a collection of independent vehicles when the objective is to climb the gradient of an environmental field. The single, heavily equipped vehicle may require considerable power to operate its sensor payload, it lacks robustness to vehicle failure and it cannot adapt the configuration or resolution of the sensor array. An independent vehicle with a single sensor may need to perform costly maneuvers to effectively climb a gradient, for instance, wandering significantly to collect rich enough data much like the "run and tumble" behavior of flagellated bacteria. In military missions, a group of autonomous vehicles are required to keep in a specified formation for area coverage and reconnaissance. In automated highway system, the throughput of the transportation network can be greatly increased if vehicles can form to platoons at a desired velocity while keeping a specified distance between vehicles. Research on formation control also helps people to better understand some biological social behaviors, such as swarm of insects and flocking of birds.

In the literature, there have been roughly three methods to formation control of multiple vehicles: leader-following, behavioral and virtual structure. Each method has its own advantages and disadvantages. In the leader-following approach, some vehicles are considered as leaders, whist the rest of robots in the group act as followers [1], [2], [3], [4]. The leaders track predefined reference trajectories, and the followers track transformed versions of the states of their nearest neighbors according to given schemes. An advantage of the leader-following approach is that it is easy to understand and implement. In addition, the formation can still be maintained even if the leader is perturbed by some disturbances. However, a disadvantage is that there is no explicit feedback to the formation, that is, no explicit feedback from the followers to the leader in this case. If the follower is perturbed, the formation cannot be maintained. Furthermore, the leader is a single point of failure for the formation. In the behavioral approach [5], [6], [7], [8], [9], [10], [11], [12], [13], [14], few desired behaviors such as collision/obstacle avoidance and goal/target seeking are prescribed for each vehicle and the formation control is calculated from a weighting of the relative importance of each behavior. The advantages of this approach are: it is natural to derive control strategies when vehicles have multiple competing objectives, and an explicit feedback is included through communication between neighbors. The disadvantages are: the group behavior cannot be explicitly defined, and it is difficult to analyze the approach mathematically and guarantee the group stability. In the virtual structure approach, the entire formation is treated as a single entity [15], [16], [17], [18]. When the structure moves, it traces out desired trajectories for each robot in the group to 
track. Some similar ideas based on the perceptive reference frame, the virtual leader, and the formation reference point are given in [14], [17], [19]. The advantages of the virtual structure approach are: it is fairly easy to prescribe the coordinated behavior for the group, and the formation can be maintained very well during the manoeuvres, i.e. the virtual structure can evolve as a whole in a given direction with some given orientation and maintain a rigid geometric relationship among multiple vehicles. However requiring the formation to act as a virtual structure limits the class of potential applications such as when the formation shape is time-varying or needs to be frequently reconfigured, this approach may not be the optimal choice. The virtual structure and leader-following approaches require that the full state of the leader or virtual structure be communicated to each member of the formation. In contrast, behavior-based approach is decentralized and may be implemented with significantly less communication. Formation feedback has been recently introduced in the literature [18], [20], [21], [22]. In [18], a coordination architecture for spacecraft formation control is introduced to incorporate the leader-following, behavioral, and virtual structure approaches to the multi-robot coordination problem. This architecture can be extended to include formation feedback. In [20], a Lyapunov formation function is used to define a formation error for a class of robots (double integrator dynamics) so that a constrained motion control problem of multiple systems is converted into a stabilization problem for one single system. The error feedback is incorporated to the virtual leader through parameterized trajectories. In terms of information from the robots in the group used for feedback in the control design for each robot, there are two main approaches to solve the problem of motion planning/control of a group of mobile robots: centralization and decentralization. In the centralized approach, see for example [18], a single controller and collision free trajectories are constructed in a workspace. The centralized approach has a drawback of computation complexity but guarantees a complete solution. The decentralized approach, see for example [23], requires less computational effort, and offers an easy way to scale the size of the robot group. This approach usually involves a combination of robot based local potential fields [14], [24], [25].

The main problem with the decentralized approach is that it is unable or extremely difficult to predict and control the critical points. Basically, the closed loop system under a controller designed by the decentralized approach has multiple equilibrium points. It is rather difficult to design a controller such that all the equilibrium points except for the desired equilibrium one are unstable/saddle points for a group of many robots. Moreover even the formation control system is designed in a centralized manner, the tuning constants in several aforementioned papers (e.g. [26], [27], [28], [29]), which are crucial to guarantee that the only desired equilibrium points are asymptotic stable and that the other critical points are unstable, are extremely difficult to obtain for practical implementation. In most of the above papers, point-robots with simple (single or double integrator) dynamics (e.g. [14], [24], [29]) or fully actuated vehicles [19] (which can be converted to a double integrator dynamics via a feedback linearization) were investigated. Vehicles with nonholonomic constraints were also considered (e.g. [5]). However, the nonholonomic kinematics are transformed to a double integrator dynamics by controlling the hand position instead of the inertial position of the vehicles. Consequently, the vehicle heading is not controlled. In addition, in the tracking control of single nonholonomic mobile robots (e.g. [30], [31], [32]) the tracking errors are often converted into special forms to deal with nonholonomic constraints using several non-trivial coordinate transformations. If these techniques are migrated to formation control of a group of nonholonomic mobile robots, it is extremely difficult to incorporate collision avoidance between the robots. The above problems motivate the contribution of this paper.

In this paper, we propose a constructive method to design a cooperative formation control system for a group of $N$ mobile robots. The simple point-mass robots are first considered to clarify the design philosophy. The proposed technique is extended to mobile robots with nonholonomic constraints (nonholonomic mobile robots). New local potential functions are constructed to design gradient based cooperative controllers to achieve almost global asymptotic convergence of a group of mobile robots to a particular formation in term of both shape and orientation, and guarantee no collisions between themselves. Formal proof of the results is given. 


\section{Problem statement}

We consider a group of $N$ simple point-mass mobile robots, of which each has the following dynamics

$$
\dot{q}_{i}=u_{i}, \quad i=1, \ldots, N
$$

where $q_{i} \in \mathrm{R}^{n}$ and $u_{i} \in \mathrm{R}^{n}$ are the state and control input of the robot $i$. We assume that $n>1$ and $N>1$. The assumption that each robot is represented as a point is not as restrictive as it may seem since various shapes can be mapped to single points through a series of transformations [26], [27], [28]. Our task is to design the control input $u_{i}$ for each robot $i$ that forces the group of $N$ robots to stabilize with respect to their group members in configurations that make a particular formation specified by a desired vector $l(\eta)=\left[l_{12}^{T}(\eta), l_{23}^{T}(\eta), \ldots, l_{N-1, N}^{T}(\eta)\right]^{T}$, where $\eta \in \mathrm{R}^{m}$ is the formation parameter vector to specify the formation change, while avoiding collisions between themselves. The parameter vector $\eta$ is used to specify rotation, expansion and contraction of the formation such that when $\eta$ converges to its desired value $\eta_{f}$, the desired shape of the formation is achieved. In addition, it requires all the robots align their velocity vectors to a desired bounded one $u_{d} \in \mathrm{R}^{n}$, and move toward specified directions specified by the desired formation velocity vector. The control objective is formally stated as follows:

Control objective: Assume that at the initial time $t_{0}$ each robot initializes at a different location, and that each robot has a different desired location, i.e. there exist strictly positive constants $\varepsilon_{1}, \varepsilon_{2}$ and $\varepsilon_{3}$ such that

$$
\begin{aligned}
& \left\|q_{i}\left(t_{0}\right)-q_{j}\left(t_{0}\right)\right\| \geq \varepsilon_{1}, \\
& \left\|l_{i j}(\eta)\right\| \geq \varepsilon_{2}, \\
& \left\|\partial l_{i j}(\eta) / \partial \eta\right\| \leq \varepsilon_{3}, \forall i, j \in\{1,2, \ldots N\}, \forall \eta \in \mathrm{R}^{m} .
\end{aligned}
$$

Design the control input $u_{i}$ for each robot $i$, and an update law for the formation parameter vector $\eta$ such that each robot (almost) globally asymptotically approaches its desired location to form a desired formation, and that the robots' velocity converges to the desired (bounded) velocity $u_{d}$ while avoiding collisions with all other robots in the group, i.e.

$$
\begin{aligned}
& \lim _{t \rightarrow \infty}\left(q_{i}(t)-q_{j}(t)-l_{i j}(\eta(t))\right)=0, \\
& \lim _{t \rightarrow \infty}\left(\eta(t)-\eta_{f}\right)=0 \\
& \lim _{t \rightarrow \infty}\left(u_{i}(t)-u_{d}\right)=0 \\
& \left\|q_{i}(t)-q_{j}(t)\right\|>\varepsilon_{4}, \forall i, j \in\{1,2, \ldots N\}, \forall t \geq t_{0} \geq 0
\end{aligned}
$$

where $\varepsilon_{4}$ is a strictly positive constant, and $\eta_{f}$ is a vector of constants that determine the desired formation. The desired formation can be represented by a labeled directed graph ([29], [34]) in the following definition.

Definition 1. The formation graph, $G=\{V, E, L\}$ is a directed labeled graph consisting of:

-a set of vertices (nodes), $V=\left\{\vartheta_{1}, \cdots, \vartheta_{N}\right\}$ indexed by the mobile robots in the group,

-a set of edges, $E=\left\{\left(\vartheta_{i}, \vartheta_{j}\right) \in V \times V\right\}$, containing ordered pairs of vertices that represent inter-robot position constraints, and

-a set of labels, $L=\left\{\gamma_{i j} \mid \gamma_{i j}=\left\|q_{i}-q_{j}-l_{i j}\right\|^{2}, \forall\left(\vartheta_{i}, \vartheta_{j}\right) \in E\right\}, l_{i j}=q_{i f}-q_{j f} \in \mathrm{R}^{n}$ indexed by the edges in $E$.

Indeed, when the control objective is achieved, the edge labels become $\left\|q_{i}-q_{j}-l_{i j}\right\|^{2}=0, \forall\left(\vartheta_{i}, \vartheta_{j}\right) \in$ $E$, i.e. the relative distance between the robots $i$ and $j$ is $l_{i j}$.

\section{Control design}

We consider the following local potential function

$$
\varphi_{i}=\gamma_{i}+\delta \beta_{i}
$$


where $\delta$ are positive tuning constants, the functions $\gamma_{i}$ and $\beta_{i}$ are the goal and related collision avoidance functions for the robot $i$ specified as follows:

-The goal function $\gamma_{i}$ is essentially the sum of all distances from the robot $i$ to its adjacent group members, $N_{i}$. A simple choice of this function is

$$
\gamma_{i}=\sum_{j \in N_{i}} \gamma_{i j}, \quad \gamma_{i j}=\frac{1}{2}\left\|q_{i}-q_{j}-l_{i j}\right\|^{2} .
$$

-The related collision function $\beta_{i}$ should be chosen such that it is equal to infinity whenever any robots come in contact with the robot $i$, i.e. a collision occurs, and attains the minimum value when the robot $i$ is at its desired location with respect to other group members belong to $N_{i}$, which are adjacent to the robot $i$. This function is chosen as follows:

$$
\beta_{i}=\sum_{j \in N_{i}}\left(\frac{\beta_{i j}^{k}}{\beta_{i j l}^{2 k}}+\frac{1}{\beta_{i j}^{k}}\right)
$$

where $k$ is a positive constant to be chosen later, $\beta_{i j}$ and $\beta_{i j l}$ are collision and desired collision functions chosen as

$$
\beta_{i j}=\frac{1}{2}\left\|q_{i}-q_{j}\right\|^{2}, \beta_{i j l}=\frac{1}{2}\left\|l_{i j}\right\|^{2} .
$$

It is noted from (7) that $\beta_{i j}=\beta_{j i}$ and $\beta_{i j l}=\beta_{j i l}$.

\section{Remark 1.}

1. The above choice of the potential function $\varphi_{i}$ given in (4) with its components specified in (5)-(6), has the following properties: 1) it attains the minimum value when the robot $i$ is at the desired location with respect to other group member belong to $N_{i}$, which are adjacent to the robot $i$, i.e. $q_{i}-q_{j}-l_{i j}=0, j \in N_{i}$, and 2) it is equal to infinity whenever one or more robots come in contact with the robot $i$, i.e. when a collision occurs.

2. The potential function (4) is different from the ones proposed in [14] and [33] in the sense that the ones in [14] and [33] are centralized and do not put penalty on the relative distance between the robots, i.e. do not include the goal function $\gamma_{i}$. Therefore, the controllers developed in [14] and [33] do not guarantee the formation converge to a specified configuration but to any configurations that locally minimize the potential functions (these potential functions in [14] and [33] are nonconvex).

3. Our potential function (4) is also different from the navigation functions proposed in [26] and [29] in the sense that our potential function is of the form of sum of collision avoidance functions while those navigation functions in [26] and [29] are of the form of product of collision avoidance functions. This feature makes our potential function "more decentralized". Furthermore, our potential function is equal to infinity while those in [14], [26] and [29] is equal to a finite constant when a collision occurs. However, those in [26] and [29] also cover obstacle and work space boundary avoidance. Although these issues are not included in this paper for clarity, considering these issues is possible and is the subject of future work.

4. Our potential function does not have problems like local minima and non-reachable goal as listed in [24].

To design the control input $u_{i}$, we differentiate both sides of (4) along the solutions of (1) to obtain

$$
\begin{aligned}
\dot{\varphi}_{i} & =\sum_{j \in N_{i}}\left[\Omega_{i j}^{T}\left(u_{i}-u_{j}\right)-\Psi_{i j}^{T} \dot{\eta}\right] \\
& =\sum_{j \in N_{i}}\left[\Omega_{i j}^{T}\left(u_{i}-u_{d}-\left(u_{j}-u_{d}\right)\right)-\Psi_{i j}^{T} \dot{\eta}\right] \\
& =\sum_{j \in N_{i}} \Omega_{i j}^{T}\left(u_{i}-u_{d}\right)-\sum_{j \in N_{i}} \Omega_{i j}^{T}\left(u_{j}-u_{d}\right)-\sum_{j \in N_{i}} \Psi_{i j}^{T} \dot{\eta}
\end{aligned}
$$


where

$$
\begin{aligned}
& \Omega_{i j}=q_{i}-q_{j}-l_{i j}+\delta k\left(\frac{1}{\beta_{i j l}^{2 k}}-\frac{1}{\beta_{i j}^{2 k}}\right) \beta_{i j}^{k-1}\left(q_{i}-q_{j}\right) \\
& \Psi_{i j}=\left[\left(q_{i}-q_{j}-l_{i j}+\frac{2 \delta k \beta_{i j}^{k}}{\beta_{i j l}^{2 k+1}} l_{i j}\right)^{T} \frac{\partial l_{i j}}{\partial \eta}\right]^{T} .
\end{aligned}
$$

From (8), we simply choose the control $u_{i}$ for the robot $i$ and the update law for $\eta$ as follows:

$$
\begin{aligned}
u_{i} & =-C \sum_{j \in N_{i}} \Omega_{i j}+u_{d} \\
\dot{\eta} & =-\Gamma\left(\eta-\eta_{f}\right)
\end{aligned}
$$

where $C \in \mathrm{R}_{+}^{n \times n}$ and $\Gamma \in \mathrm{R}_{+}^{m \times m}$ are symmetric positive definite matrices. Substituting (10) into (8) yields

$$
\dot{\varphi}_{i}=-\sum_{j \in N_{i}} \Omega_{i j}^{T} C \sum_{j \in N_{i}} \Omega_{i j}-\sum_{j \in N_{i}} \Omega_{i j}^{T}\left(u_{j}-u_{d}\right)+\sum_{j \in N_{i}} \Psi_{i j}^{T} \Gamma\left(\eta-\eta_{f}\right) .
$$

Substituting (10) into (1) results in the closed loop system

$$
\dot{q}_{i}=-C \sum_{j \in N_{i}} \Omega_{i j}+u_{d}, i=1, \ldots, N .
$$

Since the desired formation is specified in terms on relative distances between the robots, we write the closed loop system of the inter-robot dynamics from the closed loop system (12) as

$$
\dot{q}_{i j}=-C\left(\sum_{a \in N_{i}} \Omega_{i a}-\sum_{b \in N_{j}} \Omega_{j b}\right),(i, j) \in\{1, \ldots, N\}, i \neq j
$$

where $q_{i j}=q_{i}-q_{j}$. We now state the main result in the following theorem.

Theorem 1. Under the assumptions stated in the control objective, the control for each robot i given in (10) with an appropriate choice of the tuning constants $\delta$ and $k$, solves the control objective.

Proof. See Appendix.

\section{Simulations}

We carry out a simulation example in two-dimensional space to illustrate the results. The number of robots is $N=4$. The initial positions of robots are chosen randomly in the circle with a radius of 0.5 centered at the origin. The design constants are chosen as $C=\operatorname{diag}(0.4,0.4), k=0.5, \delta=0.1$. It is noted that this choice satisfies the conditions in the proof of Theorem 1. We run two simulations with $u_{d}=\left[\begin{array}{ll}1 & 0.2\end{array}\right]^{T}$ (linear formation motion meaning that each robot will move on a rectilinear line to form the desired formation) and $u_{d}=[\sin (0.5 t) \cos (0.5 t)]$ (circular formation motion meaning that each robot will move on a circle to form the desired formation). For clarity, we take the formation parameter $\eta$ as a scalar to implement formation expansion. The desired formation is depicted in Figure 1. These simulations are motivated by gradient climbing missions in which the mobile sensor network (each mobile robot serves as a mobile sensor) seeks out local maxima or minima in the environmental field. The network can adapt its configuration in response to the sensed environment in order to optimize its gradient climb. For example, gradients in temperature fields (among others) can be estimated from the data collected by the 
mobile robots; these are of interest for enabling gradient climbing to locate and track features such as fronts and eddies. These gradients can be used to compute the desired reference velocity vector $u_{d}$ in our simulations in this section. In the first 4.5 seconds (for the linear formation motion case) and 15 seconds (for the circular formation case), $\eta$ is set to zero then is updated to $\eta_{f}=3$ for the rest of simulation time. The update gain is chosen as $\Gamma=2$ (scalar).

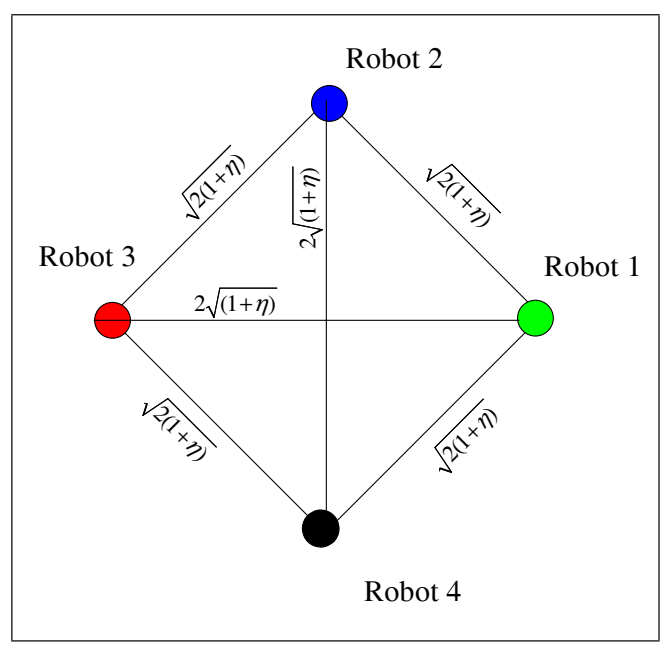

Figure 1: Desired formation for simulation.

Figures 2 and 3 plot simulation results for the linear formation motion and circular formation cases, respectively. For clarity, we only plot the control $u_{1}=\left[u_{x 1} u_{y 1}\right]^{T}$ and distances from the robot 1 to other members in the group, i.e. $\left\|q_{12}\right\|,\left\|q_{13}\right\|$ and $\left\|q_{14}\right\|$. It is seen from these figures that the desired formation shapes are nicely achieved and there are no collisions between any robots, see the bottom right figures in Figures 2 and 3, where the distances from the robot 1 to other members in the groups are plotted. Clearly, these distances are always larger than zero. It is also seen from Figures 2 and 3 that at the beginning all the robots rapidly move away from each other to avoid collisions since they start pretty close to each other.

\section{Extension to formation control of nonholonomic mobile robots}

Control of single nonholonomic mobile robots receives considerable attention, and is complicated due to the fact that they have less controls than the outputs to be controlled, see for example [30], [31], [32] and references therein. Indeed, control of a group of nonholonomic mobile robots is more complicated due to some nonholonomic (non-integral) constraint. However, in this section we show that the control method developed in Section 3 can be readily extended to force a group of $N$ nonholonomic mobile robots of unicycle type to move in such a way that a desired formation is achieved. For clarity, we consider only the kinematic model of the nonholonomic mobile robots. Designing the control system at the dynamic level even without requiring robot velocities be measured can be carried out using one more "backstepping" step [35] and our proposed exponential observer in [31]. Consider the kinematic model of the unicycle mobile robot $i$, whose only two wheels are actuated and the third wheel is not actuated 

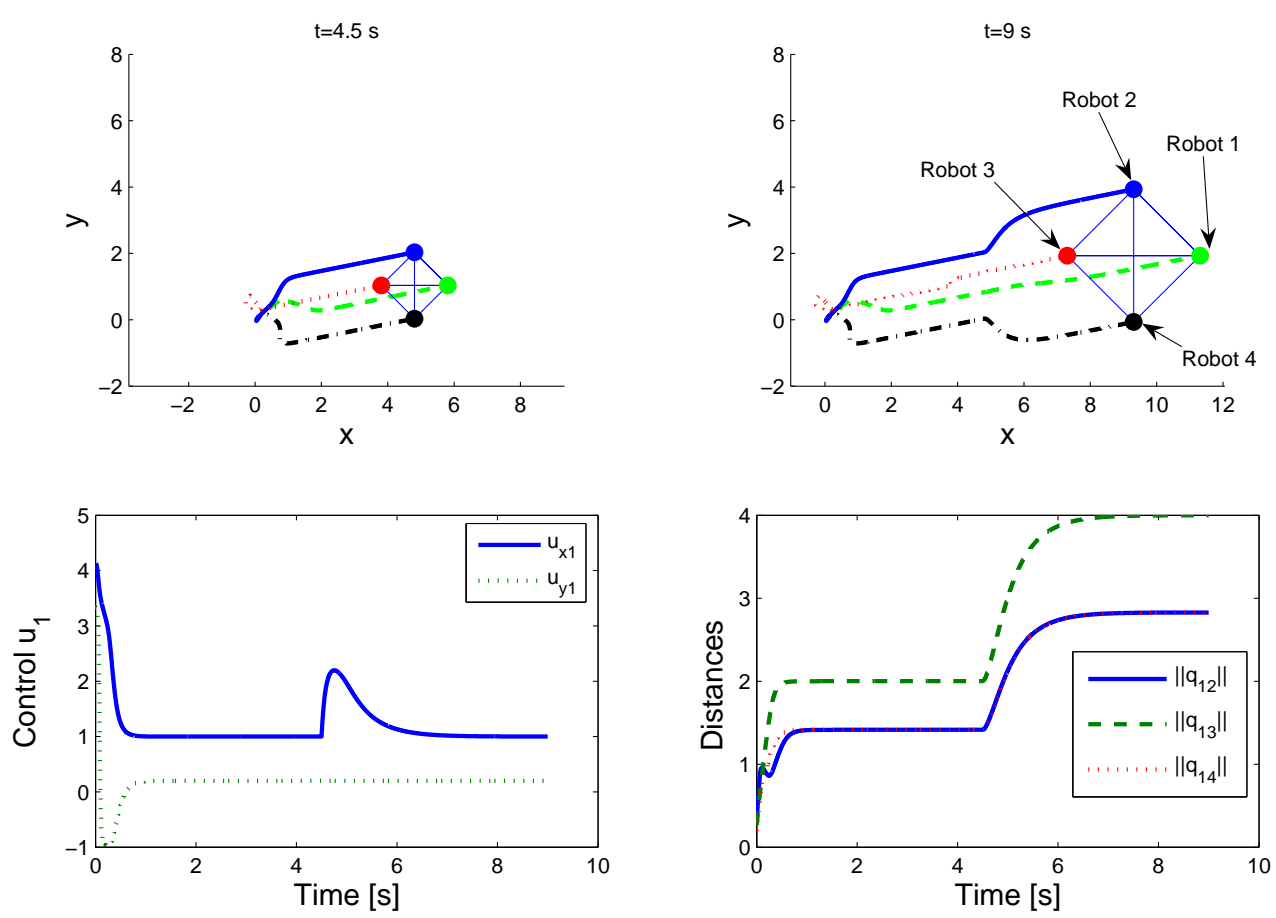

Figure 2: Linear formation motion: simulation results.

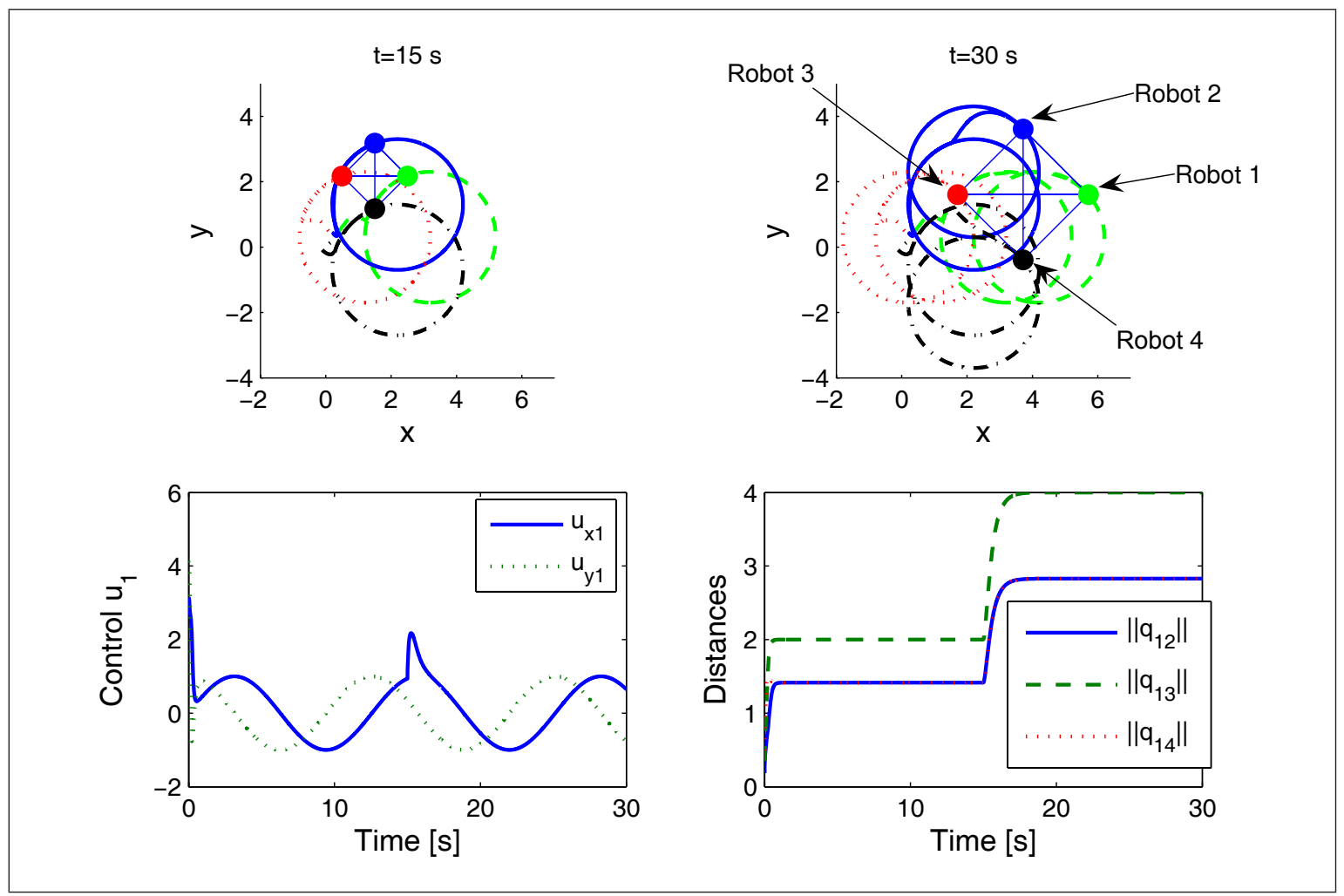

Figure 3: Circular formation motion: simulation results. 
(see Figure 4), given by

$$
\begin{aligned}
\dot{x}_{i} & =\frac{R_{i}}{2}\left(\cos \left(\theta_{i}\right) \omega_{1 i}+\cos \left(\theta_{i}\right) \omega_{2 i}\right) \\
\dot{y}_{i} & =\frac{R_{i}}{2}\left(\sin \left(\theta_{i}\right) \omega_{1 i}+\sin \left(\theta_{i}\right) \omega_{2 i}\right) \\
\dot{\theta}_{i} & =\frac{R_{i}}{2 b_{i}}\left(\omega_{1 i}-\omega_{2 i}\right)
\end{aligned}
$$

where $\left(x_{i}, y_{i}\right)$ denote the coordinates of the middle point, $P_{0 i}$, between the left and right driving wheels, and $\theta_{i}$ denotes the heading of the robot $i$ coordinated in the earth-fixed frame $O X Y$, see Figure $4, \omega_{1 i}$ and $\omega_{2 i}$ denote the angular velocities of the wheels of the robot $i$. Moreover $R_{i}$ and $b_{i}$ are defined in Figure 4. The task now is to design the control inputs $\omega_{1 i}$ and $\omega_{2 i}$ to achieve the control objective stated in Section 3. We require an additional assumption on the desired formation velocity vector $u_{d}$ that $\lim _{t \rightarrow \infty}\left\|u_{d}(t)\right\| \neq 0$, i.e. we do not consider the stabilization/regulation problem. For convenience, we convert the angular velocities of the wheels to the linear and angular velocities $\left(v_{i}\right.$ and $\left.r_{i}\right)$ of the robot $i$ by the following relationship:

$$
\left[\begin{array}{c}
v_{i} \\
r_{i}
\end{array}\right]=\left(\frac{1}{R_{i}}\left[\begin{array}{cc}
1 & b_{i} \\
1 & -b_{i}
\end{array}\right]\right)^{-1}\left[\begin{array}{c}
\omega_{1 i} \\
\omega_{2 i}
\end{array}\right]
$$

With (15), we can write (14) as

$$
\begin{aligned}
\dot{x}_{i} & =v_{i} \cos \left(\theta_{i}\right) \\
\dot{y}_{i} & =v_{i} \sin \left(\theta_{i}\right) \\
\dot{\theta}_{i} & =r_{i}
\end{aligned}
$$

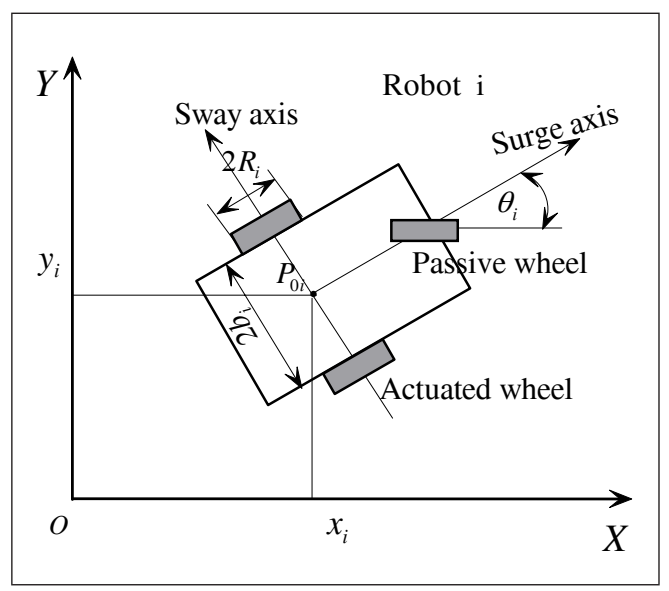

Figure 4: Geometric description of a nonholonomic mobile robot.

Indeed, the kinematic model (14) or (16) possesses the following nonholonomic constraint:

$$
\dot{x}_{i} \sin \left(\theta_{i}\right)-\dot{y}_{i} \cos \left(\theta_{i}\right)=0 .
$$

Moreover, we will consider the the linear and angular velocities $\left(v_{i}\right.$ and $\left.r_{i}\right)$ of the robot $i$ as the control inputs. After these inputs are designed, $\omega_{1 i}$ and $\omega_{2 i}$ are calculated from (15). 


\subsection{Control design}

The control design consists of two steps. At the first step, we consider the control $v_{i}$ and the yaw angle $\theta_{i}$ as a virtual control to steer the robot position $\left(x_{i}, y_{i}\right)$ to its desired location. At the second step, the control $r_{i}$ will be desired to force the virtual yaw angle to converge to its actual yaw angle.

Step 1. Define

$$
\theta_{e i}=\theta_{i}-\alpha_{\theta_{i}}
$$

where $\alpha_{\theta_{i}}$ is a virtual control of $\theta_{i}$. With (18), we can write (16) as

$$
\dot{q}_{i}=u_{i}+\Lambda_{\theta_{e i}}
$$

where

$$
q_{i}=\left|\begin{array}{c}
x_{i} \\
y_{i}
\end{array}\right|, u_{i}=\left|\begin{array}{c}
\cos \left(\alpha_{\theta_{i}}\right) \\
\sin \left(\alpha_{\theta_{i}}\right)
\end{array}\right| v_{i}, \Lambda_{\theta_{e i}}=\left|\begin{array}{c}
\left(\cos \left(\theta_{e i}\right)-1\right) \cos \left(\alpha_{\theta_{i}}\right)-\sin \left(\theta_{e i}\right) \sin \left(\alpha_{\theta_{i}}\right) \\
\sin \left(\theta_{e i}\right) \cos \left(\alpha_{\theta_{i}}\right)+\left(\cos \left(\theta_{e i}\right)-1\right) \sin \left(\alpha_{\theta_{i}}\right)
\end{array}\right| v_{i} .
$$

It is seen that (19) is almost of the same form as (1). However, the problem is that the controls $v_{i}$ and $\alpha_{\theta_{i}}$ are not solvable directly from the control $u_{i}$ if $u_{i}$ is not designed properly. We therefore present briefly how $u_{i}$ is designed to tackle that problem. Consider the following potential function (the same form as (4))

$$
\varphi_{i}=\gamma_{i}+\delta \beta_{i}
$$

where $\delta, \gamma_{i}$ and $\beta_{i}$ are defined in Section 3, see (6) and (7). Differentiating both sides of (21) along the solutions of (19) gives

$$
\dot{\varphi}_{i}=\sum_{j \in N_{i}}\left[\Omega_{i j}^{T}\left(u_{i}+\Lambda_{\theta_{e i}}-u_{d}^{*}-\left(u_{j}+\Lambda_{\theta_{e j}}-u_{d}^{*}\right)\right)-\Psi_{i j}^{T} \dot{\eta}\right]
$$

where $\Omega_{i j}$ and $\Psi_{i j}$ are defined in (9), and $u_{d}^{*}=\sqrt{1+\sum_{i=1}^{N}\left\|\sum_{j \in N_{i}} \Omega_{i j}\right\|^{2}} u_{d}$. It is noted that we use $u_{d}^{*}$ instead of $u_{d}$ in (22) to overcome the nonholonomic problem of the mobile robot under investigation. Indeed, $\lim _{t \rightarrow \infty} \sum_{j \in N_{i}} \Omega_{i j}(t)=0$ implies that $\lim _{t \rightarrow \infty} u_{d}^{*}(t)=u_{d}$. From (22), we choose the control $u_{i}$ and the update law for $\eta$ as

$$
\begin{aligned}
& u_{i}=-C\left\|u_{d}\right\| \sum_{j \in N_{i}} \Omega_{i j}+u_{d}^{*} \\
& \dot{\eta}=-\Gamma\left(\eta-\eta_{f}\right)
\end{aligned}
$$

where $C$ and $\Gamma$ are diagonal positive definite matrices. Again, $\left\|u_{d}\right\|$ is included in the control $u_{i}$ to overcome the nonholonomic problem. Defining $\theta_{d}=\arctan \left(u_{d y} / u_{d x}\right)$, then from the first equations of (23) and (20), we have

$$
\begin{aligned}
& \cos \left(\alpha_{\theta_{i}}\right) v_{i}=-c_{1}\left\|u_{d}\right\| \sum_{j \in N_{i}} \Omega_{x i j}+\sqrt{1+\sum_{i=1}^{N}\left\|\sum_{j \in N_{i}} \Omega_{i j}\right\|^{2}}\left\|u_{d}\right\| \cos \left(\theta_{d}\right) \\
& \sin \left(\alpha_{\theta_{i}}\right) v_{i}=-c_{2}\left\|u_{d}\right\| \sum_{j \in N_{i}} \Omega_{y i j}+\sqrt{1+\sum_{i=1}^{N}\left\|\sum_{j \in N_{i}} \Omega_{i j}\right\|^{2}}\left\|u_{d}\right\| \sin \left(\theta_{d}\right)
\end{aligned}
$$

where $\Omega_{x i j}$ and $\Omega_{y i j}$ are defined as $\Omega_{i j}=\left[\Omega_{x i j} \Omega_{y i j}\right]^{T}, c_{1}$ and $c_{2}$ are defined as $C=\operatorname{diag}\left(c_{1}, c_{2}\right)$. We now need to solve (24) for $v_{i}$ and $\alpha_{\theta_{i}}$. To do this, multiplying both sides of the first and second equations of (24) with $\cos \left(\theta_{d}\right)$ and $\sin \left(\theta_{d}\right)$, respectively, then adding them together result in

$$
\begin{aligned}
\cos \left(\alpha_{\theta_{i}}-\theta_{d}\right) v_{i}= & -c_{1}\left\|u_{d}\right\| \sum_{j \in N_{i}} \Omega_{x i j} \cos \left(\theta_{d}\right)- \\
& c_{2}\left\|u_{d}\right\| \sum_{j \in N_{i}} \Omega_{y i j} \sin \left(\theta_{d}\right)+\sqrt{1+\sum_{i=1}^{N}\left\|\sum_{j \in N_{i}} \Omega_{i j}\right\|^{2}\left\|u_{d}\right\| .}
\end{aligned}
$$


On the other hand, multiplying both sides of the first and second equations of (24) with $\sin \left(\theta_{d}\right)$ and $\cos \left(\theta_{d}\right)$, respectively, then subtracting from each other result in

$$
\sin \left(\alpha_{\theta_{i}}-\theta_{d}\right) v_{i}=c_{1}\left\|u_{d}\right\| \sum_{j \in N_{i}} \Omega_{x i j} \sin \left(\theta_{d}\right)-c_{2}\left\|u_{d}\right\| \sum_{j \in N_{i}} \Omega_{y i j} \cos \left(\theta_{d}\right) .
$$

From (25) and (26), we have

$$
\alpha_{\theta_{i}}=\theta_{d}+\arctan \left(\frac{c_{1} \sum_{j \in N_{i}} \Omega_{x i j} \sin \left(\theta_{d}\right)-c_{2} \sum_{j \in N_{i}} \Omega_{y i j} \cos \left(\theta_{d}\right)}{-c_{1} \sum_{j \in N_{i}} \Omega_{x i j} \cos \left(\theta_{d}\right)-c_{2} \sum_{j \in N_{i}} \Omega_{y i j} \sin \left(\theta_{d}\right)+\sqrt{1+\sum_{i=1}^{N}\left\|\sum_{j \in N_{i}} \Omega_{i j}\right\|^{2}}}\right) .
$$

It is seen that (27) is well-defined if the positive constants $c_{1}$ and $c_{2}$ are chosen such that

$$
c_{1}+c_{2}<1
$$

The control $v_{i}$ is found by solving (24) as

$$
\begin{gathered}
v_{i}=\cos \left(\alpha_{\theta_{i}}\right)\left\|u_{d}\right\|\left(-c_{1} \sum_{j \in N_{i}} \Omega_{x i j}+\sqrt{1+\sum_{i=1}^{N}\left\|\sum_{j \in N_{i}} \Omega_{i j}\right\|^{2}} \cos \left(\theta_{d}\right)\right)+ \\
\sin \left(\alpha_{\theta_{i}}\right)\left\|u_{d}\right\|\left(-c_{2} \sum_{j \in N_{i}} \Omega_{y i j}+\sqrt{1+\sum_{i=1}^{N}\left\|\sum_{j \in N_{i}} \Omega_{i j}\right\|^{2}} \sin \left(\theta_{d}\right)\right) .
\end{gathered}
$$

Substituting (23) into (22) results in

$$
\dot{\varphi}_{i}=-\left\|u_{d}\right\| \sum_{j \in N_{i}} \Omega_{i j}^{T} C \sum_{j \in N_{i}} \Omega_{i j}+\sum_{j \in N_{i}}\left[\Omega_{i j}^{T}\left(\Lambda_{\theta_{e i}}-\left(u_{j}+\Lambda_{\theta_{e j}}-u_{d}^{*}\right)\right)+\Psi_{i j}^{T} \Gamma\left(\eta-\eta_{f}\right)\right] .
$$

Step 2. To design the control $r_{i}$, differentiating both sides of (18) along the solutions of the third equation of (16) and choosing the control $r_{i}$ as

$$
r_{i}=-d_{i} \theta_{e i}-\dot{\alpha}_{\theta_{i}}-\sum_{j \in N_{i}} \Omega_{i j}^{T} \Lambda_{\theta_{e i}} / \theta_{e i}
$$

where $d_{i}$ is a positive constant, and the term $\sum_{j \in N_{i}} \Omega_{i j}^{T} \Lambda_{\theta_{e i}} / \theta_{e i}$ is to cancel the cross term $\sum_{j \in N_{i}} \Omega_{i j}^{T} \Lambda_{\theta_{e i}}$ in (30), result in

$$
\dot{\theta}_{e i}=-d_{i} \theta_{e i}-\sum_{j \in N_{i}} \Omega_{i j}^{T} \Lambda_{\theta_{e i}} / \theta_{e i}
$$

Note that $\Lambda_{\theta_{e i}} / \theta_{e i}$ is well defined $\operatorname{since} \sin \left(\theta_{i e}\right) / \theta_{i e}=\int_{0}^{1} \cos \left(\theta_{i e} \lambda\right) d \lambda$ and $\left(\cos \left(\theta_{i e}\right)-1\right) / \theta_{i e}=\int_{0}^{1} \sin \left(\theta_{i e} \lambda\right) d \lambda$ are smooth functions.

\subsection{Stability analysis}

We consider the following function

$$
\varphi_{t o t}=\log \left(1+\sum_{i=1}^{N}\left(\varphi_{i}+\theta_{e i}^{2}\right)\right)+\frac{1}{2}\left(\eta-\eta_{f}\right)^{T} \Gamma\left(\eta-\eta_{f}\right)
$$

whose derivative along the solutions of (30), (32) and the second equation of (23) satisfies

$$
\dot{\varphi}_{t o t}=-2 \frac{\left\|u_{d}\right\| \sum_{i=1}^{N} \sum_{j \in N_{i}} \Omega_{i j}^{T} C \sum_{j \in N_{i}} \Omega_{i j}+\sum_{i=1}^{N} d_{i} \theta_{e i}^{2}}{1+\sum_{i=1}^{N}\left(\varphi_{i}+\theta_{e i}^{2}\right)}+\frac{\sum_{i=1}^{N} \sum_{j \in N_{i}} \Psi_{i j}^{T} \Gamma\left(\eta-\eta_{f}\right)}{1+\sum_{i=1}^{N}\left(\varphi_{i}+\theta_{e i}^{2}\right)}-\left(\eta-\eta_{f}\right)^{T} \Gamma\left(\eta-\eta_{f}\right)
$$


where we have used

$$
\begin{aligned}
-\sum_{i=1}^{N} \sum_{j \in N_{i}}\left[\Omega_{i j}^{T}\left(u_{j}+\Lambda_{\theta_{e j}}-u_{d}^{*}\right)\right. & =\sum_{i=1}^{N} \sum_{j \in N_{i}}\left[\Omega_{i j}^{T}\left(u_{i}-u_{d}^{*}+\Lambda_{\theta_{e i}}\right)\right. \\
& =-\left\|u_{d}\right\| \sum_{i=1}^{N} \sum_{j \in N_{i}} \Omega_{i j}^{T} C \sum_{j \in N_{i}} \Omega_{i j}+\sum_{i=1}^{N} \sum_{j \in N_{i}} \Omega_{i j}^{T} \Lambda_{\theta_{e i}} .
\end{aligned}
$$

The rest of stability analysis can be carried out in the same lines as in Proof of Theorem 1 since (34) is of the same form as (40) and $\lim _{t \rightarrow \infty}\left\|u_{d}(t)\right\| \neq 0$ by assumption. Finally, note that $\lim _{t \rightarrow \infty} \theta_{e i}(t)=0$ and $\lim _{t \rightarrow \infty} \sum_{j \in N_{i}} \Omega_{i j}(t)=0$ implies that $\lim _{t \rightarrow \infty}\left(\theta_{i}(t)-\theta_{d}\right)=0$, i.e. the yaw angle of all robots converge to the desired angle $\theta_{d}=\arctan \left(u_{d y} / u_{d x}\right)$.

\subsection{Simulation results}

We now perform a simulation to illustrate the results in the previous subsection. The number of robots, initial conditions of the robot positions, control gains, desired formation velocity and desired formation shape are the same as in Section 4. The robot heading angles are initialized randomly in the circle with a radius of 0.5 centered at the origin. For clarity, we only simulate the circular formation motion, and we do not include simulation results on the formation expansion as in Section 4, i.e. the formation parameter $\eta$ is set to zero in all the simulation time. The other design constants are chosen as $d_{i}=5$. Simulation results are plotted in Figure 5. Again, it is seen that the robots are forced to move to nicely achieve the desired formation and no collisions between the robots occur. Moreover, the yaw angle of all robots converges to the desired value $\theta_{d}$, see the top-right figure in Figure 5, where the yaw angle errors are plotted. A close look at Figure 5 shows that the main different between simulation results in this subsection and those in Section 4 is that the robots take a longer time to approach the desired formation. This is because we use the heading angles $\theta_{i}$ as the virtual controls to steer the robots to overcome the noholonomic constraint.

\section{Summary and Conclusions}

This paper has contributed the method to construct local potential functions, based on which gradientlike cooperative controllers were designed for a group of mobile robots both with and without nonholonomic constraints to perform certain formation missions. Formal analysis of the convergence and feasibility of the control solutions have also been provided. In the near future, it is of interest to apply the proposed control design method combined with the control design scheme for other single underactuated robots with second order nonholonomic constraints such as underactuated ships [37] to achieve a desired formation for a group of underactuated systems.

\section{Appendix: Proof of Theorem 1}

We prove Theorem 1 in two steps. At the first step, we show that there are no collisions between any robots and the solutions of the closed loop system exist. At the second step, we prove that the equilibrium point of the inter-robot dynamics closed loop system (13), at which $q_{i}-q_{j}-l_{i j}=0$, is asymptotically stable. Finally, we show that all other equilibrium(s) of (13) are either unstable or saddle.

Step 1. Proof of no collision and existence of solutions:

We consider the following common potential function $\varphi$ given by

$$
\varphi=\sum_{i=1}^{N} \varphi_{i}
$$




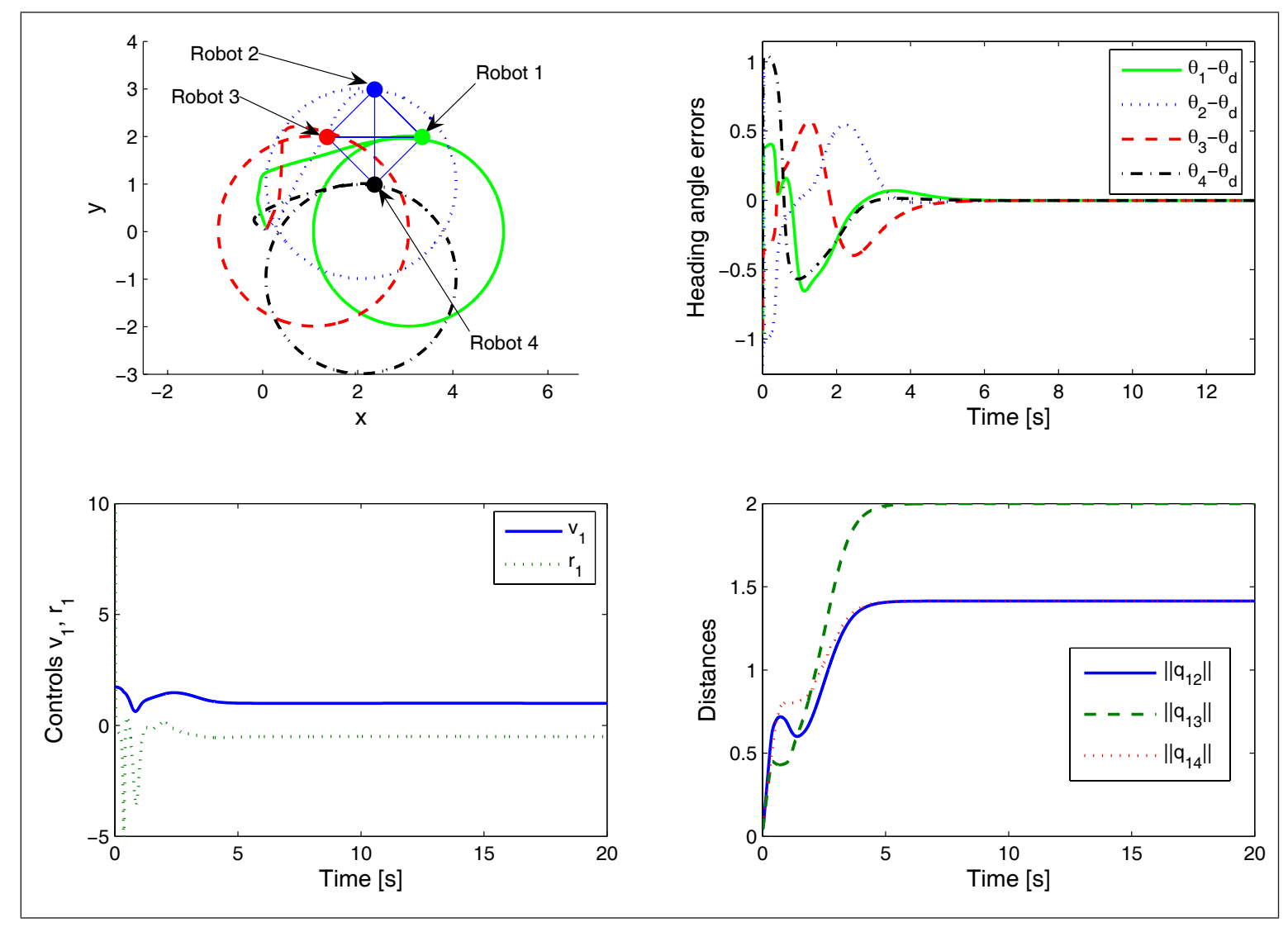

Figure 5: Mobile robot circular formation motion: simulation result. 
whose derivative along the solutions of (11) is

$$
\dot{\varphi}=-\sum_{i=1}^{N} \sum_{j \in N_{i}} \Omega_{i j}^{T} C \sum_{j \in N_{i}} \Omega_{i j}-\sum_{i=1}^{N} \sum_{j \in N_{i}} \Omega_{i j}^{T}\left(u_{j}-u_{d}\right)+\sum_{i=1}^{N} \sum_{j \in N_{i}} \Psi_{i j}^{T} \Gamma\left(\eta-\eta_{f}\right) .
$$

Since $l_{i j}=-l_{j i}$ and $\Omega_{i j}=-\Omega_{j i}$, we have

$$
\sum_{i=1}^{N} \sum_{j \in N_{i}} \Omega_{i j}^{T}\left(u_{j}-u_{d}\right)=-\sum_{i=1}^{N} \sum_{j \in N_{i}} \Omega_{i j}^{T}\left(u_{i}-u_{d}\right) .
$$

Substituting (38) into (37) gives

$$
\dot{\varphi}=-2 \sum_{i=1}^{N} \sum_{j \in N_{i}} \Omega_{i j}^{T} C \sum_{j \in N_{i}} \Omega_{i j}+\sum_{i=1}^{N} \sum_{j \in N_{i}} \Psi_{i j}^{T} \Gamma\left(\eta-\eta_{f}\right) .
$$

We now consider the following total function $\varphi_{t o t}=\log (1+\varphi)+0.5\left\|\eta-\eta_{f}\right\|^{2}$ whose derivative along the solutions of (39) the second equation of (10) satisfies

$$
\dot{\varphi}_{t o t}=-\frac{2}{1+\varphi} \sum_{i=1}^{N} \sum_{j \in N_{i}} \Omega_{i j}^{T} C \sum_{j \in N_{i}} \Omega_{i j}+\frac{1}{1+\varphi} \sum_{i=1}^{N} \sum_{j \in N_{i}} \Psi_{i j}^{T} \Gamma\left(\eta-\eta_{f}\right)-\left(\eta-\eta_{f}\right)^{T} \Gamma\left(\eta-\eta_{f}\right)
$$

which implies that

$$
\begin{aligned}
\dot{\varphi}_{\text {tot }} \leq \quad & -\frac{2}{1+\varphi} \sum_{i=1}^{N} \sum_{j \in N_{i}} \Omega_{i j}^{T} C \sum_{j \in N_{i}} \Omega_{i j}+\frac{\lambda_{\max }(\Gamma)}{4 \varepsilon(1+\varphi)^{2}}\left\|\sum_{i=1}^{N} \sum_{j \in N_{i}} \Psi_{i j}^{T}\right\|^{2}- \\
& \left(\lambda_{\min }(\Gamma)-\varepsilon \lambda_{\max }(\Gamma)\right)\left\|\eta-\eta_{f}\right\|^{2}
\end{aligned}
$$

where $\varepsilon$ is a positive constant, $\lambda_{\min }(\Gamma)$ and $\lambda_{\max }(\Gamma)$ denote the minimum and maximum eigenvalues of $\Gamma$ respectively. From (9), and definition of the function $\varphi$, it can be readily shown that there exists a positive constant $\omega_{\max }$ such that

$$
\frac{1}{(1+\varphi)^{2}}\left\|\sum_{i=1}^{N} \sum_{j \in N_{i}} \Psi_{i j}^{T}\right\|^{2} \leq \omega_{\max }
$$

With (42) in mind, picking $\varepsilon=\lambda_{\min }(\Gamma) / \lambda_{\max }(\Gamma)$ we can write (41) as

$$
\dot{\varphi}_{t o t} \leq \frac{\lambda_{\max }^{2}(\Gamma)}{4 \lambda_{\min }(\Gamma)} \omega_{\max } \triangleq \varpi_{\max }
$$

Integrating both sides of (43) results in

$$
\varphi_{\text {tot }}(t) \leq \varphi_{t o t}\left(t_{0}\right)+\varpi_{\max }\left(t-t_{0}\right)
$$

where $\varphi_{t o t}(t)$ and $\varphi_{t o t}\left(t_{0}\right)$ are (from the definition of $\varphi_{t o t}$ )

$$
\begin{aligned}
& \varphi_{\text {tot }}(t)=\log \left[1+\sum_{i=1}^{N}\left(\gamma_{i}(t)+\delta \sum_{j \in N_{i}}\left(\frac{\beta_{i j}^{k}(t)}{\beta_{i j l}^{2 k}}+\frac{1}{\beta_{i j}^{k}(t)}\right)\right)\right]+\frac{1}{2}\left\|\eta(t)-\eta_{f}\right\|^{2} \\
& \varphi_{\text {tot }}\left(t_{0}\right)=\log \left[1+\sum_{i=1}^{N}\left(\gamma_{i}\left(t_{0}\right)+\delta \sum_{j \in N_{i}}\left(\frac{\beta_{i j}^{k}\left(t_{0}\right)}{\beta_{i j l}^{2 k}}+\frac{1}{\beta_{i j}^{k}\left(t_{0}\right)}\right)\right)\right]+\frac{1}{2}\left\|\eta\left(t_{0}\right)-\eta_{f}\right\|^{2} .
\end{aligned}
$$

The right hand side of (44) cannot escape to infinity unless when $t=\infty$ since $\beta_{i j l}>0$ and $\beta_{i j}\left(t_{0}\right)>0$ (see definition of $\beta_{i j l}$ and $\beta_{i j}$ given in (7)). Therefore the left hand side of (44) cannot escape to infinity for 
all $t \in\left[t_{0}, \infty\right)$. This implies that $\beta_{i j}(t)$ cannot be zero for all $t \in\left[t_{0}, \infty\right)$, i.e. no collisions can occur for all $t \in\left[t_{0}, \infty\right)$. On the other hand, it is true from the second equation of (10) that

$$
\left\|\eta(t)-\eta_{f}\right\| \leq\left\|\eta\left(t_{0}\right)-\eta_{f}\right\| e^{-\lambda_{\min }(\Gamma)\left(t-t_{0}\right)}
$$

which means that the desired formation shape is achieved exponentially. Using (42) and (46), we can write (41) as

$$
\dot{\varphi}_{t o t} \leq \lambda_{\max }(\Gamma) \sqrt{\omega_{\max }}|| \eta\left(t_{0}\right)-\eta_{f} \| e^{-\lambda_{\min }(\Gamma)\left(t-t_{0}\right)} .
$$

Integrating both sides of (47) from $t_{0}$ to $t$ results in

$$
\varphi_{t o t}(t) \leq \varphi_{t o t}\left(t_{0}\right)+\lambda_{\max }(\Gamma) \sqrt{\omega_{\max }}\left\|\eta\left(t_{0}\right)-\eta_{f}\right\| / \lambda_{\min }(\Gamma) .
$$

It is seen that the right hand side of (48) is bounded. Therefore the left hand side of (48) must also be bounded. This implies that $\beta_{i j}(t)$ must be larger than a strictly positive constant for all $t \in\left[t_{0}, \infty\right)$, which in turn means that there exists a strictly positive constant $\varepsilon_{4}$ such that the last inequality of (3) holds. To prove that the solutions of the closed loop system (12) exist, we consider the function $W=$ $0.5 \sum_{i=1}^{N}\left\|q_{i}\right\|^{2}$ whose derivative along the solutions of (12), after some simple manipulation, satisfies $\dot{W} \leq$ $\rho_{1}\left(1+1 / \min \left(\beta_{i j}\right)\right) W+\rho_{2}$, where $\rho_{1}$ and $\rho_{2}$ are some positive constants, which implies that the solutions of (12) exist since $\beta_{i j}(t)$ is larger than a strictly positive constant for all $t \in\left[t_{0}, \infty\right)$. Furthermore, applying Barbalat's lemma found in [36] to (41) gives

$$
\lim _{t \rightarrow \infty} \frac{1}{1+\varphi(t)} \sum_{i=1}^{N} \sum_{j \in N_{i}} \Omega_{i j}^{T}(t) C \sum_{j \in N_{i}} \Omega_{i j}(t)=0
$$

which implies that

$$
\left\{\begin{array} { l } 
{ \operatorname { l i m } _ { t \rightarrow \infty } \sum _ { j \in N _ { i } } \Omega _ { i j } ( t ) = 0 } \\
{ \operatorname { l i m } _ { t \rightarrow \infty } \varphi ( t ) = \chi _ { 1 } }
\end{array} \text { or } \left\{\begin{array}{l}
\lim _{t \rightarrow \infty} \sum_{j \in N_{i}} \Omega_{i j}(t)=\chi_{2} \\
\lim _{t \rightarrow \infty} \varphi(t)=\infty
\end{array}\right.\right.
$$

where $\chi_{1}$ and $\chi_{2}$ are some constants. From definitions of $\Omega_{i j}$ and $\varphi$, the second limit set in (50) cannot be true. Therefore, the first limit set in (50) implies that $\lim _{t \rightarrow \infty} \sum_{j \in N_{i}} \Omega_{i j}(t)=0$.

Step 2. Behavior near equilibrium points.

At the steady state, the equilibrium points are found by solving the following equations

$$
\sum_{j \in N_{i}} \Omega_{i j}=\sum_{j \in N_{i}}\left(q_{i j}-l_{i j}+\delta k\left(\frac{1}{\beta_{i j l}^{2 k}}-\frac{1}{\beta_{i j}^{2 k}}\right) \beta_{i j}^{k-1} q_{i j}\right)=0, i=1, \ldots, N .
$$

It is directly verified that $\bar{q}=\bar{l}$ where $\bar{q}$ and are stack vectors of $q_{i j}$ and $l_{i j}$, respectively, i.e. $\bar{q}=$ $\left[q_{12}^{T}, q_{13}^{T} \ldots, q_{N-1, N}^{T}\right]^{T}$ and $\bar{l}=\left[l_{12}^{T}, l_{13}^{T} \ldots, l_{N-1, N}^{T}\right]^{T}$, is one root of (51). In addition there is (are) another $\operatorname{root}(\mathrm{s})$ denoted by $\bar{q}_{c}=\left[q_{12 c}^{T}, q_{13 c}^{T} \ldots, q_{N-1, N c}^{T}\right]^{T}$ of (51) different from $\bar{l}$ satisfying

$$
\left.\sum_{j \in N_{i}} \Omega_{i j}\right|_{\bar{q}=\bar{q}_{c}}=\sum_{j \in N_{i}}\left(q_{i j c}-l_{i j}+\delta k\left(\frac{1}{\beta_{i j l}^{2 k}}-\frac{1}{\beta_{i j c}^{2 k}}\right) \beta_{i j c}^{k-1} q_{i j c}\right)=0, i=1, \ldots, N
$$

where $\beta_{i j c}=0.5\left\|q_{i c}-q_{j c}\right\|^{2}$. In the following, we will show that the equilibrium point $\bar{q}=\bar{l}$ is asymptotically stable, and the equilibrium point(s) $\bar{q}=\bar{q}_{c}$ is (are) unstable or saddle. We now write the closed loop system of the inter-robot dynamics (13) as

$$
\dot{\bar{q}}=-\bar{C} F(\bar{q}, \bar{l}) .
$$


where $\bar{C}=\operatorname{diag}(\underbrace{C, \cdots, C}_{E})$ with $E$ the number of edges of the formation graph, and

$$
\begin{aligned}
F(\bar{q}, \bar{l})=\quad & {\left[\sum_{a \in N_{1}} \Omega_{1 a}^{T}-\sum_{b \in N_{2}} \Omega_{2 b}^{T}, \sum_{a \in N_{1}} \Omega_{1 a}^{T}-\sum_{b \in N_{3}} \Omega_{3 b}^{T}, \ldots, \sum_{a \in N_{i}} \Omega_{i a}^{T}-\sum_{b \in N_{j}} \Omega_{j b}^{T}, \ldots .\right.} \\
& \left.\sum_{a \in N_{N-1}} \Omega_{N-1, a}^{T}-\sum_{b \in N_{N}} \Omega_{N b}^{T}\right]^{T} .
\end{aligned}
$$

Since (52) holds for all $i=1, \ldots, N$, at the steady state we have $\sum_{a \in N_{i}} \Omega_{i a}-\sum_{b \in N_{j}} \Omega_{j b}=0, \forall(i, j) \in\{1, \ldots, N\}, i \neq$ $j$. Therefore the equilibrium points $\bar{q}=\bar{l}$ and $\bar{q}=\bar{q}_{c}$ are also the equilibrium points of (53). The general gradient of $F(\bar{q}, \bar{l})$ with respect to $\bar{q}$ is given by

$$
\begin{gathered}
\frac{\partial F(\bar{q}, \bar{l})}{\partial \bar{q}}=\left[\begin{array}{cccc}
\frac{\partial \Xi_{12}}{\partial q_{12}} & \frac{\partial \Xi_{12}}{\partial q_{13}} & \cdots & \frac{\partial \Xi_{12}}{\partial q_{N-1, N}} \\
\vdots & \vdots & \vdots & \vdots \\
\frac{\partial \Xi_{i j}}{\partial q_{12}} & \cdots & \frac{\partial \Xi_{i j}}{\partial q_{i j}} & \frac{\partial \Xi_{i j}}{\partial q_{N-1, N}} \\
\vdots & \vdots & \vdots & \vdots \\
\frac{\partial \Xi_{N-1, N}}{\partial q_{12}} & \cdots & \cdots & \frac{\partial \Xi_{N-1, N}}{\partial q_{N-1, N}}
\end{array}\right], \\
\Xi_{i j}=\sum_{a \in N_{i}} \Omega_{i a}-\sum_{b \in N_{j}} \Omega_{j b},(i, j) \in\{1, \ldots, N\}, i \neq j .
\end{gathered}
$$

It can be checked that

$$
\begin{aligned}
& \frac{\partial \Xi_{i j}}{\partial q_{i j}}=N I_{n \times n}+2 \delta k\left(\frac{1}{\beta_{i j l}^{2 k}}-\frac{1}{\beta_{i j}^{2 k}}\right) \beta_{i j}^{k-1} I_{n \times n}+2 \delta k\left((k-1)\left(\frac{1}{\beta_{i j l}^{2 k}}-\frac{1}{\beta_{i j}^{2 k}}\right) \beta_{i j}^{k-2}+\frac{2 k}{\beta_{i j}^{k+2}}\right) q_{i j} q_{i j}^{T} \triangleq H_{i j} \\
& \frac{\partial \Xi_{i j}}{\partial q_{c d}}=s \delta k\left(\frac{1}{\beta_{c d l}^{2 k}}-\frac{1}{\beta_{c d}^{2 k}}\right) \beta_{c d}^{k-1} I_{n \times n}+s \delta k\left((k-1)\left(\frac{1}{\beta_{c d l}^{2 k}}-\frac{1}{\beta_{c d}^{2 k}}\right) \beta_{c d}^{k-2}+\frac{2 k}{\beta_{c d}^{k+2}}\right) q_{c d} q_{c d}^{T},
\end{aligned}
$$

where $(c, d) \in\{1, \ldots, N\},(c, d) \neq(i, j), c \neq d$, and $s=1$ or $s=-1$ depending on value of $c, d, i$ and $j$. However, we do not need to specify the sign of $s$ for our next task. We now investigate properties of the equilibrium points $\bar{q}=\bar{l}$ and $\bar{q}=\bar{q}_{c}$ based on the general gradient $\partial F(\bar{q}, \bar{l}) / \partial \bar{q}$ evaluated at those points.

Step 2.1 Proof of $\bar{q}=\bar{l}$ being the asymptotic stable equilibrium point:

At the equilibrium point $\bar{q}=\bar{l}$, we have

$$
\left.\frac{\partial \Xi_{i j}}{\partial q_{i j}}\right|_{\bar{q}=\bar{l}}=N I_{n \times n}+\frac{4 \delta k^{2}}{\beta_{i j l}^{k+2}} l_{i j} l_{i j}^{T},\left.\quad \frac{\partial \Xi_{i j}}{\partial q_{c d}}\right|_{\bar{q}=\bar{l}}=s \frac{2 \delta k^{2}}{\beta_{c d l}^{k+2}} l_{c d} l_{c d}^{T},
$$

where $\beta_{c d l}=0.5\left\|l_{c d}\right\|^{2}$. With (57), let $\xi \in \mathrm{R}^{n E}$ we have

$$
\left.\xi^{T} \frac{\partial F(\bar{q}, \bar{l})}{\partial \bar{q}}\right|_{\bar{q}=\bar{l}} \xi \geq\left(N-\frac{4 \delta k^{2} n E \max \left(l_{i j a}^{2}\right)}{\min \left(\beta_{i j l}^{k+2}\right)}\right) \xi^{T} \xi, \quad(i, j) \in\{1, \ldots, N\}, i \neq j
$$

where $l_{i j a}$ is the $a^{\text {th }}$ element of $l_{i j}$. Therefore, for any given constant $k$ if we choose the tuning constant $\delta$ such that

$$
N-\frac{4 \delta k^{2} n E \max \left(l_{i j a}^{2}\right)}{\min \left(\beta_{i j l}^{k+2}\right)}>0 \rightarrow \delta<\frac{N \min \left(\beta_{i j l}^{k+2}\right)}{4 k^{2} n E \max \left(l_{i j a}^{2}\right)},(i, j) \in\{1, \ldots, N\}, i \neq j
$$

then the matrix $\partial F(\bar{q}, \bar{l}) /\left.\partial \bar{q}\right|_{\bar{q}=\bar{l}}$ is positive definite, which in turn implies that the equilibrium point $\bar{q}=\bar{l}$ is asymptotically stable. 
Step 2.2. Proof of $\bar{q}=\bar{q}_{c}$ being the unstable/saddle equilibrium point(s): The idea is to consider block matrices on the main diagonal of the matrix $\partial F(\bar{q}, \bar{l}) /\left.\partial \bar{q}\right|_{\bar{q}=\bar{q}_{c}}$ and show that there exists at least one block matrix whose determinant is negative. Define $H_{i j c}=\partial \Xi_{i j} /\left.\partial q_{i j}\right|_{\bar{q}=\bar{q}_{c}}$ and let $\phi_{a}$ and $\phi_{b}$ be the $a^{t h}$ and $b^{t h}$ elements of $q_{i j c},(a, b) \in\{1, \ldots, n\}, a \neq b$. We form the matrices $H_{i j c}^{a b}$ from the matrix $H_{i j c}$ as follows

$$
\begin{aligned}
& H_{i j c}^{a b}=\left[\begin{array}{ll}
h_{11} & h_{12} \\
h_{21} & h_{22}
\end{array}\right] \\
& h_{11}=N+2 \delta k \Pi_{i j c} \beta_{i j c}^{k-1}+2 \delta k\left[(k-1) \Pi_{i j c} \beta_{i j c}^{k-2}+2 k / \beta_{i j c}^{k+2}\right] \phi_{a}^{2} \\
& h_{12}=2 \delta k\left[(k-1) \Pi_{i j c} \beta_{i j c}^{k-2}+2 k / \beta_{i j c}^{k+2}\right] \phi_{a} \phi_{b} \\
& h_{21}=2 \delta k\left[(k-1) \Pi_{i j c} \beta_{i j c}^{k-2}+2 k / \beta_{i j c}^{k+2}\right] \phi_{a} \phi_{b} \\
& h_{22}=N+2 \delta k \Pi_{i j c} \beta_{i j c}^{k-1}+2 \delta k\left[(k-1) \Pi_{i j c} \beta_{i j c}^{k-2}+2 k / \beta_{i j c}^{k+2}\right] \phi_{b}^{2}
\end{aligned}
$$

where $\Pi_{i j c}=1 / \beta_{i j l}^{2 k}-1 / \beta_{i j c}^{2 k}$. The determinant of $H_{i j c}^{a b}$ is given by

$$
\operatorname{det}\left(H_{i j c}^{a b}\right)=\left(N+2 \delta k \Pi_{i j c} \beta_{i j c}^{k-1}\right) \Delta_{i j c}^{a b}
$$

where

$$
\Delta_{i j c}^{a b}=N+2 \delta k \Pi_{i j c} \beta_{i j c}^{k-1}+2 \delta k\left[(k-1) \Pi_{i j c} \beta_{i j c}^{k-2}+2 k / \beta_{i j c}^{k+2}\right]\left(\phi_{a}^{2}+\phi_{b}^{2}\right)
$$

Let us consider the sum:

$$
\sum_{a=1}^{n-1} \sum_{b=a+1}^{n} \Delta_{i j c}^{a b}=n(n-1) N+2 \delta k(n-1)(2(k-1)+n) \beta_{i j c}^{k-1} / \beta_{i j l}^{2 k}+2 \delta k(n-1)(2(k+1)-n) / \beta_{i j c}^{k+1} .
$$

Since $n>1$, picking $k>n / 2-1$ ensures that $\sum_{a=1}^{n-1} \sum_{b=a+1}^{n} \Delta_{i j c}^{a b}>0$. Therefore, there exists at least one pair $(a, b) \in\{1, \ldots, n\}$ denoted by $\left(a^{*}, b^{*}\right)$ such that $\Delta_{i j c}^{a^{*} b^{*}}>0$. Now for all $(i, j) \in\{1, \ldots, N\}, i \neq j$ let us consider the sum:

$$
\sum_{i=1}^{N-1} \sum_{j=i+1}^{N} \frac{\operatorname{det}\left(H_{i j c}^{a^{*} b^{*}}\right)}{\Delta_{i j c}^{a^{*} b^{*}}} \beta_{i j c}=\sum_{i=1}^{N-1} \sum_{j=i+1}^{N}\left(N \beta_{i j c}+2 \delta k \Pi_{i j c} \beta_{i j c}^{k}\right) .
$$

On the other hand, multiplying both sides of $F\left(\bar{q}_{c}, \bar{l}\right)=0$ with $\bar{q}_{c}^{T}$ results in $\bar{q}_{c}^{T} F\left(\bar{q}_{c}, \bar{l}\right)=0$, which is expanded to

$$
\sum_{i=1}^{N-1} \sum_{j=i+1}^{N}\left(N q_{i j c}^{T}\left(q_{i j c}-l_{i j}\right)+2 \delta k N \Pi_{i j c} \beta_{i j c}^{k}\right)=0 .
$$

Substituting (65) into (64) results in

$$
\sum_{i=1}^{N-1} \sum_{j=i+1}^{N} \frac{\operatorname{det}\left(H_{i j c}^{a^{*} b^{*}}\right)}{\Delta_{i j c}^{a^{*} b^{*}}} \beta_{i j c}=\sum_{i=1}^{N-1} \sum_{j=i+1}^{N}(N-2) \beta_{i j c}+\sum_{i=1}^{N-1} \sum_{j=i+1}^{N} q_{i j c}^{T} l_{i j}
$$

The term $\sum_{i=1}^{N-1} \sum_{j=i+1}^{N}\left(q_{i j c}^{T} l_{i j}\right)$ is strictly negative since at the point where $q_{i j}=l_{i j}$ (the point $F$ in Figure 6) all attractive and repulsive forces are equal to zero while at the point where $q_{i j}=q_{i j c}$ (the point $C$ in Figure 6) the sum of attractive and repulsive forces is equal to zero (see Section 2 for discussion of a simple case). Therefore the point $q_{i j}=0$ (the point $O$ in Figure 6) must locate between the points $q_{i j}=l_{i j}$ and $q_{i j}=q_{i j c}$, see Figure 6. Furthermore if we write (65) as

$$
2 \sum_{i=1}^{N-1} \sum_{j=i+1}^{N} \beta_{i j c}+\delta k\left(\beta_{i j c}^{k} / \beta_{i j l}^{2 k}-1 / \beta_{i j c}^{k}\right)=\sum_{i=1}^{N-1} \sum_{j=i+1}^{N} q_{i j c}^{T} l_{i j}
$$




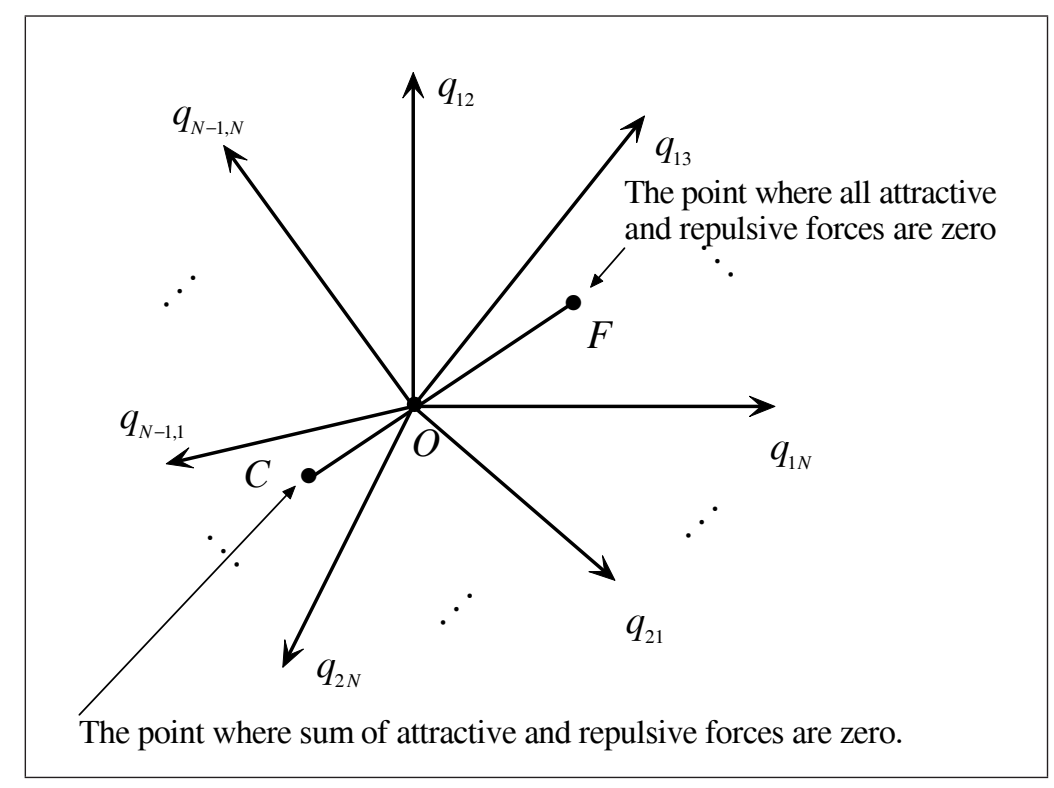

Figure 6: Illustration of location of critical points.

we can see that deceasing $\delta$ results in decrease in $\beta_{i j c}$ since $\beta_{i j l}$ is a bounded constant and the right hand side of (67) is negative. Therefore, choosing a sufficiently small $\delta$ ensures that the right hand of (64) is strictly negative since $\beta_{i j c}=0.5\left\|q_{i j c}\right\|^{2}$. That is

$$
\sum_{i=1}^{N-1} \sum_{j=i+1}^{N} \frac{\operatorname{det}\left(H_{i j c}^{a^{*} b^{*}}\right)}{\Delta_{i j c}^{a^{*} b^{*}}} \beta_{i j c}<0
$$

which implies that there exists at least one pair $(i, j) \in\{1, \ldots, N\}$ denoted by $\left(i^{*}, j^{*}\right)$ such that

$$
\operatorname{det}\left(H_{i^{*} j^{*} c}^{a^{*} b^{*}}\right)<0
$$

The inequality implies that at least one eigenvalue of the matrix $\partial F(\bar{q}, \bar{l}) /\left.\partial \bar{q}\right|_{\bar{q}=\bar{q}_{c}}$ is negative. This in turn guarantees that $\bar{q}_{c}$ is an unstable/saddle equilibrium point of (53). Proof of Theorem 1 is completed.

\section{References}

[1] P. K. C. Wang, Navigation Strategies for Multiple Autonomous Mobile Robots Moving in Formation, J. Robot. Syst., Vol. 8, No. 2, pp. 177-195, 1991.

[2] P. K. C. Wang and F. Y. Hadaegh, Coordination and Control of Multiple Microspacecraft Moving in Formation, J. Astronautical Sci., Vol. 44, No. 3, pp. 315-355, 1996.

[3] J. P. Desai, J. Ostrowski, and V. Kumar, Controlling Formations of Multiple Mobile Robots, Proc. IEEE Int. Conf. Robotics and Automation, Leuven, Belgium, pp. 2864-2869, 1998.

[4] M. Mesbahi and F. Y. Hadaegh, Formation Flying Control of Multiple Spacecraft via Graphs, Matrix Inequalities, and Switching, AIAA J. Guidance, Control, Dynam., Vol. 24, no. 2, pp. 369-377, 2000.

[5] R. T. Jonathan, R. W. Beard and B. J. Young, A Decentralized Approach to Formation Maneuvers, IEEE Trans. Robot. and Automat., Vol. 19, pp. 933-941, 2003. 
[6] T. D. Barfoot and C. M. Clark, Motion Planning for Formations of Mobile Robots, Robot. Auton. Syst., vol. 46, pp. 65-78, 2004.

[7] H. Yamaguchi, Adaptive Formation Control for Distributed Autonomous Mobile Robot Groups, Proc. IEEE Int. Conf. Robotics and Automation, Albuquerque, NM, pp. 2300-2305, 1997.

[8] T. Balch and R. C. Arkin, Behavior-Based Formation Control for Multirobot Teams, IEEE Trans. Robot. Automat., Vol. 14, pp. 926-939, 1998.

[9] M. Schneider-Fontan and M. J. Mataric, Territorial Multirobot Task Division, IEEE Trans. Robot. Automat., Vol. 14, pp. 815-822, 1998.

[10] Q. Chen and J. Y. S. Luh, Coordination and Control of a Group of Small Mobile Robots, Proc. IEEE Int. Conf. Robotics and Automation, pp. 2315-2320, 1994.

[11] M. Veloso, P. Stone, and K. Han, The CMUnited-97 Robotic Soccer Team: Perception and Multiagent Control, Robot. Auton. Syst., Vol. 29, pp. 133-143, 1999.

[12] L. E. Parker, ALLIANCE: An Architecture for Fault-tolerant Multirobot Cooperation, IEEE Trans. Robot. Automat., Vol. 14, pp. 220-240, 1998.

[13] K. Sugihara and I. Suzuki, Distributed Algorithms for Formation of Geometric Patterns with Many Mobile Robots, J. Robot. Syst., Vol. 13, no. 3, pp. 127-139, 1996.

[14] N. E. Leonard and E. Fiorelli, Virtual Leaders, Artificial Potentials and Coordinated Control of Groups, Proc. IEEE Conf. Decision and Control, Orlando, FL, pp. 2968-2973, 2001.

[15] W. Kang, N. Xi, and A. Sparks, Formation Control of Autonomous Agents in 3D Workspace, Proc. IEEE Int. Conf. Robotics and Automation, San Francisco, CA, pp. 1755-1760, 2000.

[16] M. A. Lewis and K.-H. Tan, High Precision Formation Control of Mobile Robots Using Virtual Structures, Auton. Robots, Vol. 4, pp. 387-403, 1997.

[17] W. Kang and H.-H. Yeh, Coordinated Attitude Control of Multisatellite Systems, Int. J. Robust Nonlinear Control, Vol.12, pp. 185-205, 2002.

[18] R. W. Beard, J. Lawton, and F. Y. Hadaegh, A Coordination Architecture for Formation Control, IEEE Trans. Control Syst. Technol., Vol. 9, pp. 777-790, 2002.

[19] R. Skjetne, S. Moi and T. I. Fossen, Nonlinear Formation Control of Marine Craft, Proc. IEEE Conf. on Decision and Control, Las Vegas, NV, pp. 1699-1704, 2002.

[20] P. Ogren, M. Egerstedt and X. Hu, A Control Lyapunov Function Approach to Multiagent Coordination, IEEE Trans. Robot. Autom. Vol.18, pp. 847-851.

[21] W. Ren and R. W. Beard, Formation Feedback Control for Multiple Spacecraft via Virtual Structures. IEE Proceedings-Control Theory Application, Vol. 151, pp. 357-368, 2004.

[22] E. W. Jush and P. S. Krishnaprasad, Equilibria and Steering Laws for Planar Formations, Syst. Contr. Letters, Vol. 52, pp. 25-38, 2004.

[23] D. M. Stipanovica, G. Inalhana, R. Teo and C. J. Tomlina, Decentralized overlapping control of a formation of unmanned aerial vehicles, Automatica, vol. 40, pp. 1285-1296, 2004.

[24] S. S. Ge and Y. J. Cui, New Potential Functions for Mobile Robot Path Planning, IEEE Trans. Robot. Automat., Vol. 16, pp. 615-620, 2000. 
[25] S. S. Ge and Y. J. Cui, Dynamics Motion Planning for Mobile Robots using Potential Field Method, Auton. Robots, Vol. 13, pp. 207-222, 2000.

[26] E. Rimon and D.E. Koditschek, Exact Robot Navigation Using Artificial Potential Functions, IEEE Trans. Robot. and Automat., Vol. 8, no. 5, pp. 501-518, 1992.

[27] E. Rimon and D.E. Koditschek, Robot Navigation Functions on Manifolds with Boundary, Advances in Applied Mathematics, Vol. 11, pp. 412-442, 1990.

[28] H. G. Tanner, S. G. Loizou and K. J. Kyriakopoulos, Nonholonomic Navigation and Control of Multiple Mobile Robot Manipulators, IEEE Trans. Robot. Automat., Vol. 19, no. 1, pp. 53-64, 2003.

[29] H.G. Tanner and A. Kumar, Towards Decentralization of Multi-robot Navigation Functions, IEEE Int. Conf. Robotics and Automation, Barcelona, Spain, pp 4143-4148, 2005.

[30] W. E. Dixon, D. M. Dawson, E. Zergeroglu and A. Behal, Nonlinear Control of Wheeled Mobile Robots, Springer, London, 2001.

[31] K. D. Do, Z. P. Jiang and J. Pan, A Global Output-feedback Controller for Simultaneous Tracking and Stabilization of Mobile Robots, IEEE Trans. Robot. Automat., Vol.20, pp. 589-584, 2004.

[32] T. Fukao, H. Nakagawa, and N. Adachi, Adaptive tracking control of nonholonomic mobile robot, IEEE Trans. Robot. Automat., Vol. 16, pp. 609-615, 2000.

[33] H. G. Tanner, A. Jadbabaie and G. J. Pappas, Stable Flocking of Mobile Agents, Part I: Fixed Topology, Proc. IEEE Conf. Decision and Control, Hawaii, pp. 2010-2015, 2003.

[34] G. Godsils and G. Royle, Algebraic Graph Theory, New York: Springer Graduate Texts in Mathematics, 207, 2001.

[35] M. Krstic, I. Kanellakopoulos and P.V. Kokotovic, Nonlinear and Adaptive Control Design, New York: Wiley, 1995.

[36] H. Khalil, Nonlinear Systems, Prentice Hall, 2002.

[37] K. D. Do and J. Pan, Global Path-tracking of Underactuated Ships with Non-zero Off-diagonal Terms, Automatica, Vol. 41, pp. 87-95, 2005.

Dang Binh Nguyen Thai Nguyen University of Technology

Rector's Office

3-2 Street, Thai Nguyen City, Vietnam E-mail: nguyen.d.binh.tnut@gmail.com

Khac Duc Do

School of Mechanical Engineering

The University of Western Australia 35 Stirling Highway, Crawley, WA 6009, Australia E-mail: duc@mech.uwa.edu.au 